\title{
Relación entre Inteligencia Emocional y salud mental en Orientadores Educativos
}

\author{
Javier Cejudo
}

Departamento de Psicología, Universidad de Castilla La Mancha, Ciudad Real

\section{España}

Correspondencia: Javier Cejudo. Facultad de Educación de Ciudad Real, Ronda de Calatrava, 3 13071, Ciudad Real. España. E-mail: manueljavier.cejudo@uclm.es

(C) Education \& Psychology I+D+i and Ilustre Colegio Oficial de la Psicología de Andalucía Oriental (Spain) 


\section{Resumen}

Introducción. El objetivo de la presente investigación estuvo dirigido a estudiar la relación de la inteligencia emocional, como capacidad, y la inteligencia emocional, como rasgo con salud mental, en una muestra de orientadores educativos.

Método. La muestra ha estado compuesta de 203 orientadores. Los instrumentos utilizados han sido: Mayer-Salovey-Caruso Emotional Intelligence Test (MSCEIT), Trait Emotional Intelligence Questionnaire Short Form (TEIQUE-SF) y la Escala de Salud Mental (MH5). En primer lugar, se analizaron las correlaciones entre las puntuaciones correspondientes a todas las variables. Posteriormente se estudiaron las diferencias de medias ( $t$ de Student) en función de la etapa educativa y en función del sexo. Finalmente, con el objetivo de explorar las variables que predicen la salud mental se realizó un análisis de regresión lineal múltiple, paso a paso.

Resultados. Los resultados evidencian que las relaciones existentes entre la IE como rasgo y la salud mental son más elevadas que las relaciones entre IE como capacidad y salud mental. Por otro lado, se constatan puntuaciones significativamente más elevadas en los orientadores de Educación Secundaria en la variable de inteligencia emocional como capacidad en comprensión emocional. También se constatan puntuaciones significativamente más elevadas en la variable salud mental en los orientadores de Educación Primaria. Igualmente, se constatan puntuaciones significativamente más elevadas en las mujeres en la puntuación de la inteligencia emocional como capacidad en regulación emocional y puntuaciones significativamente más elevadas en la puntuación de la inteligencia emocional como rasgo de personalidad en los hombres. Por último, el análisis de regresión múltiple ha permitido identificar como variable predictora de la salud mental, la inteligencia emocional como rasgo.

Discusión. El presente estudio supone uno de los primeros trabajos desarrollados en una muestra de orientadores educativos en los que se analiza la influencia de la inteligencia emocional como rasgo y la inteligencia emocional como capacidad, sobre la salud mental. Además aporta evidencias de validez predictiva de la IE como rasgo sobre un indicador de salud mental.

Palabras Clave: inteligencia emocional capacidad, inteligencia emocional rasgo, salud mental, orientadores educativos. 


\title{
Relationship between Emotional Intelligence and mental health in School Counselors
}

\begin{abstract}
Introduction: The purpose of the present research is aimed at studying the relationship between emotional intelligence as an ability and emotional intelligence as a trait and mental health of a sample of school counsellors.

Method: The sample has been made up of 203 school counsellors. The instruments used have been: Mayer-Salovey-Caruso Emotional Intelligence Test (MSCEIT), Trait Emotional Intelligence Questionnaire Short Form (TEIQUE-SF) and the Mental Health Scale (MH-5). In the first place, the correlations among the scoring corresponding to all of the variables were analised. Subsequently, the differences in average ( $t$ of Student) were studied according to the educational stage in which the school counsellors are working and according to their sex. Finally, with the aim of exploring the variables that predict mental health, an analysis of multiple lineal regression was carried out, step by step.

Results: The results show that the existing relationships between EI as a trait and mental health are higher than those existing between EI as an ability and mental health. On the other hand, scorings significantly higher were registered in the school counsellors in Secondary School in the variable of emotional intelligence as an ability in emotional comprehension. Scorings significantly higher are also registered in the variable of mental health of school counsellors in Primary Education. Likewise, scorings significantly higher are also registered in women in their score of emotional intelligence as an ability in emotional regulation, and scorings significantly higher in the score of emotional intelligence as a personality trait in men. Finally, the analysis of multiple regression has allowed for identifying emotional intelligence as a trait as a predictor variable of mental health.
\end{abstract}

Discussion: The present study represents one of the first research papers carried out in a sample of school counsellors in which the influence on mental health of emotional intelligence as a trait and as an ability is analised. Furthermore, it provides evidence about the predictive validity of EI as a trait over an indicator of mental health.

Keywords: emotional intelligence ability, emotional intelligence trait, mental health, school counselors. 


\section{Introducción}

Tal y como recoge Pérez-González (2010) la orientación educativa se concibe como un factor de calidad de la educación (Martínez, Quintanal y Téllez, 2002), cuya finalidad se debe centrar en favorecer el desarrollo integral de la personalidad de todo el alumnado (Grañeras y Parras, 2008).

\section{Problemática de la Orientación Educativa}

Según Bisquerra (2006) la Orientación Educativa es un proceso de ayuda y acompamiento continuo a todas las personas en todas sus esferas, con el objetivo de potenciar la prevención y el desarrollo humano a lo largo de toda la vida. Esta ayuda se realiza mediante una intervención profesionalizada, fundamentada en principios científicos. Asimismo, según Rodríguez-Moreno (1995) el proceso de orientación educativa sería, fundamentalmente, guiar, conducir, indicar de manera procesual para ayudar a las personas a conocerse a sí mismas y al mundo que les rodea; es auxiliar a un individuo a clarificar la esencia de su vida, a comprender que él es una unidad con significado capaz de y con derecho a usar de su libertad, de su dignidad personal, dentro de un clima de igualdad de oportunidades y actuando en calidad de ciudadano responsable, tanto en su actividad laboral como en su tiempo libre.

En este sentido, y en consonancia con los planteamientos de Bisquerra (2006) la mayoría de definiciones de orientación educativa ponen el acento en el caracter de ayuda como rasgo definitorio. Es necesario destacar que la orientación se concibe como un proceso continuo, que debe ser considerada como parte integrante del proceso educativo, que implica a todos los educadores y que debe llegar a todas las personas, en todos sus aspectos de desarrollo personal y durante todo el ciclo vital. Concebimos la orientación como una intervención para lograr unos objetivos determinados enfocados preferentemente hacia la prevención, el desarrollo humano y la intervención social. Dentro del desarrollo se incluye el autodesarrollo, es decir, la capacidad de desarrollarse a sí mismo como consecuencia de la autoorientación. Esto significa que la orientación se dirige hacia el desarrollo de la autonomía personal como una forma de educar para la vida.

En este contexto es necesario generar un debate en la comunidad educativa sobre los cambios legislativos, formativos, e identitarios necesarios para que la escuela verdaderamente contribuya al desarrollo integral de la personalidad del alumnado, y además sería una buena 
ocasión para reflexionar sobre la inclusión de las competencias sociales y emocionales de forma explícita en el sistema escolar. Compartimos la idea con Extremera y FernándezBerrocal (2004) sobre la necesaria implicación de la escuela y la administración en la asunción de este reto, pero es ineludible facilitar a los educadores una formación de calidad y especialmente a los orientadores educativos. Por lo tanto, en nuestra opinión, la escuela tiene en el presente siglo XXI la responsabilidad de educar las emociones de nuestros hijos tanto o más que la propia familia (Extremera y Fernández-Berrocal, 2004).

En este sentido, los orientadores de los centros educativos tienen una importante responsabilidad en el diseño, desarrollo y evaluación de iniciativas para la mejora de las escuelas y debe asumir un papel de líder educativo capacitado para asumir competencias de liderazgo y acompañar al equipo directivo en la consecución del que abogamos, objetivo máximo de la escuela, el desarrollo integral de la personalidad del alumnado. Por ello, proponemos concebir al orientador como un agente educativo en compromiso con la mejora del centro que colabora con los docentes para mejorar el desarrollo del alumnado de manera integral, trabajando en estrecho vínculo con el equipo directivo y promoviendo la innovación en la práctica diaria.

En este orden de ideas, es necesario resumir las funciones y tareas que ha de realizar el orientador en su labor diaria:

1) Asesorar a los docentes para la mejora del proceso de enseñanza-aprendizaje.

2) Implicar y motivar a todos los miembros de la comunidad educativa para la mejora de la institución educativa.

3) Asesorar al profesorado y al equipo directivo sobre estrategias para la mejora de la convivencia en el centro y la resolución de problemas dentro y fuera del aula.

4) Potenciar en el centro iniciativas innovadoras que propicien la mejora de los procesos de enseñanza-aprenzaje, implicando a todos los profesionales del centro educativo.

5) Asesorar a los miembros del equipo directivo en su papel de líderes educativos e impulsores de todas las medidas necesarias para la mejora del centro.

6) Apoyar al estudiante en su desarrollo educativo, emocional y profesional.

Por lo tanto, es necesaria la capacitación profesional de los orientadores, tanto en su formación inicial como en su formación continua, a nivel pedagógico, psicológico, estratégico 
y mediador que le permita desempeñar su trabajo, con garantías de calidad, en los centros educativos. Asimismo pensamos que la figura del orientador es relevante para hacer realidad el cambio interno en la escuela y el liderazgo se concibe como un aspecto constitutivo de la identidad de un orientador educativo.

Es obvio que las funciones, tareas y responsabilidades anteriormente expuestas necesitan competencias de carácter técnico-profesional pero también competencias de carácter sociopersonal. Según Bisquerra y Pérez (2007) estas competencias de carácter técnicoprofesional tienen que ver con conocimientos y procedimientos en relación a un determinado ámbito profesional o de especialización. Tienen que ver con el saber y el saber hacer indispensables para el desempeño experto de una actividad laboral. Mientras que las competencias socio-personales están relacionados con conocimientos y procedimientos intrapersonales (e.g. automotivación) e interpersonales (e.g. resolución de conflictos).

En este punto es necesario aclarar que la organización de los servicios de orientación en España presenta peculiaridades en función de las normativas que realizan las diferentes administraciones educativas regionales. En este sentido, y puesto que el presente estudio de investigación se circunscribe a la comunidad autónoma de Castilla La Mancha (España) es necesario explicar el modelo de orientación de dicha comunidad autónoma. La orientación educativa y profesional se desarrollará en los centros docentes sostenidos con fondos públicos no universitarios desde un modelo de orientación mixto, estructurado en tres niveles de intervención, los dos de primeros de carácter interno y el último de carácter externo al centro: a. En un primer nivel, a través de la tutoría, coordinada por el tutor del grupo, con la colaboración de todo el profesorado. b. En un segundo nivel, por los equipos de orientación y apoyo en centros de Educación Infantil y Primaria y por los departamentos de orientación en los centros de educación secundaria, coordinada por los orientadores que forman parte de estas estructuras. c. En un tercer nivel, por los equipos de orientación técnica que se determinen, para garantizar una respuesta más especializada al alumnado que presente necesidades educativas especiales asociadas a discapacidad motora, auditiva, trastornos del desarrollo y la conducta, o altas capacidades.

\section{El debate en torno a la inteligencia emocional}

El avance científico más importante en el estudio de estas competencias sociopersonales se han generado en el marco del estudio de la inteligencia emocional. La inteligen- 
cia emocional ha suscitado un gran interés en el ámbito educativo como una vía para mejorar el desarrollo socioemocional de los alumno y como una característica fundamental en los docentes.

El concepto inteligencia emocional (IE) apareció por primera vez desarrollado en 1990 en un artículo publicado por Peter Salovey y John Mayer. Aunque su difusión se debió fundamentalmente a la publicación del libro Inteligencia emocional (1995) por parte de Daniel Goleman, psicólogo y periodista americano. La premisa fundamental de los planteamientos de Goleman (1995) es la necesidad de generar un nuevo marco de estudio de la inteligencia humana más allá de los aspectos cognitivos e intelectuales que resalte la importancia del uso y gestión del mundo emocional y social para comprender el curso de la vida de las personas.

No obstante, compartimos la idea de que "la venta de la IE como una panacea para los males del mundo ha sido severamente criticada, y es ampliamente aceptado que las pretenciosas afirmaciones que siguieron el libro de Goleman no tienen credibilidad" (Roberts, Zeidner, y Matthews, 2007, p. 430). Además autores como Davies, Stankov y Roberts (1998) defienden que la IE es un concepto difuso, cuyo estatus empírico resulta aún cuestionable (ya que, aún existen graves problemas para su medición objetiva y en cuanto a su fiabilidad), pero no por ello, carente de interés, sobre todo, en la medida que se encuentren procedimientos adecuados para estimarla y se consolide su identidad específica.

Tal y como recogen Bisquerra, Pérez-González y García (2015) la categorización conceptual más admitida en IE distingue entre modelos mixtos y modelos de capacidad que están basados en el procesamiento de la información (Mayer, Salovey y Caruso, 2000). FernándezBerrocal y Extremera (2006, pp. 67-68) exponen que "el modelo mixto es una visión muy amplia que concibe la IE como un conjunto de rasgos estables de personalidad, competencias socio-emocionales, aspectos motivacionales y diversas habilidades cognitivas (Bar-On, 2000; Goleman, 1995). El modelo de capacidad es una visión más restringida defendida por autores como Salovey y Mayer que conciben la IE como una inteligencia genuina basada en el uso adaptativo de las emociones y su aplicación a nuestro pensamiento.

En este sentido, coincidimos con, Pérez, Petrides y Furham, (2007, p. 83) en que defienden que la distinción de Mayer, Caruso y Salovey (2000) entre modelos mixtos versus 
modelos de capacidad no concuerda con la teoría psicométrica aceptada hoy en día, dado que descuida el aspecto del método de medida, ni tampoco concuerda con toda la evidencia empírica disponible hasta el momento, la cual muestra claramente que las medidas de autoinforme de IE tienden a intercorrelacionar fuertemente entre sí, independientemente de si están basadas o no en modelos mixtos o de capacidad. Todos los datos que se vienen publicando al respecto continúan poniendo de relieve la necesidad de distinguir entre dos constructos de IE, a tener en cuenta: IE como rasgo de personalidad e IE como capacidad (O'Connor y Little, 2003).

Además Pérez-González, Petrides y Furnham (2007, p. 82) añaden que "la distinción entre modelos mixtos y modelos de capacidad no tiene en cuenta el aspecto más transcendental de la operacionalización de un constructo (por ejemplo, el método de medida), y es perfectamente compatible con la idea de que las capacidades cognitivas se puedan evaluar a través de autoinformes (Mayer, Caruso y Salovey, 2000)".

La IE como capacidad se ha concebido como "una capacidad para percibir, asimilar, comprender y regular las propias emociones y la de los demás promoviendo un crecimiento emocional e intelectual" (Mayer y Salovey, 1997, p.10). La IE como capacidad debería ser medida a través de pruebas de rendimiento máximo o de ejecución máxima (Cronbach, 1972). Mientras que la IE como rasgo se referiría a "una constelación de disposiciones comportamentales y autopercepciones concernientes a las capacidades propias para reconocer, procesar y utilizar las informaciones con carga emocional, que son evaluadas a través de autoinformes y que se hallan en un nivel de generalidad inferior al que ostentan grandes factores de personalidad de segundo orden como los 5 grandes" (Petrides y Furnham, 2003; Petrides, Frederickson y Furnham, 2004). La IE como rasgo debería ser medida mediante pruebas de rendimiento típico o de ejecución típica (Cronbach, 1972). Además la relación entre la IE como capacidad y la IE como rasgo es sólo moderada (Joseph y Newman, 2010).

En este marco teórico y después de 25 años del estudio de la IE podemos concluir que ha cobrado un gran interés entre la comunidad científica por sus potenciales implicaciones beneficiosas en diversos ámbitos tales como el ámbito de la salud y del bienestar, en el ámbito educativo o en el ámbito organizacional. 
Tal y como recogen Schutte, Malouff y Thorsteinsson (2013) muchos estudios empíricos proporcionan información con respecto a las variables asociadas con la IE como rasgo y la IE como capacidad. Podemos destacar algunos meta- análisis (Malouff, Thorsteinsson, Bhullar, y Rooke, 2007; Martins, Ramalho, y Morin, 2010) que han resumido la relación entre una mayor IE y una mejor salud mental y física a través de numerosos estudios. Según el metaanálisis de Martins et al. (2010) que incluyó a 19.000 participantes y concluyó que existían asociaciones significativas de la inteligencia emocional con la salud mental, la salud psicosomática y salud física. También es reseñable en el área de las relaciones sociales, los resultados obtenidos en un meta-análisis con 1.188 participantes que concluye que existe unaasociación significativa entre una mayor IE y una mayor satisfacción en la relación de pareja (Malouff, Schutte y Thorsteinsson, 2012). Además en el ámbito organizacional un meta-análisis sobre IE y rendimiento laboral (Joseph y Newman, 2010) reportaron una asociación entre puntuaciones elevadas de IE capacidad y un mejor rendimiento laboral e igualmente una asociación entre puntuaciones elevadas en IE como rasgo y un mejor rendimiento laboral. Estos resultados entre IE y rendimiento laboral también son corroborados por otro meta-análisis realizado por O'Boyle, Humphrey, Polack, Hawver, y Story (2011). Igualmente, MartínezGonzález, Piqueras y Ramos- Linares (2010) recogen que altos niveles de IE están relacionados con una buena salud mental.

\section{Objetivos e hipótesis}

El presente estudio está dirigido a estudiar la IE como capacidad y como rasgo de personalidad de los orientadores educativos y su relación con la salud mental de una muestra de orientadores educativos de la Comunidad Autónoma de Castilla La Mancha. En esta línea han sido objetivos concretos del estudio los siguientes:

1) Analizar la relación existente entre IE como capacidad e IE como rasgo.

2) Analizar la relación existente entre IE como capacidad y salud mental.

3) Analizar la relación existente entre IE como rasgo y salud mental.

4) Estudiar las posibles diferencias existentes en IE como capacidad y como rasgo de personalidad y en la salud mental en función de la etapa educativa en la que actualmente ejercen los orientadores educativos participantes.

5) Estudiar las posibles diferencias existentes en IE como capacidad y como rasgo de personalidad y en salud mental en función del sexo de los orientadores educativos participantes. 
6) Identificar variables predictoras (IE como capacidad o IE como rasgo) de la salud mental.

De manera consecuente las hipótesis de investigación fueron las siguientes:

H1: Existirán relaciones moderadas entre IE como capacidad e IE como rasgo de personalidad.

H2: Las relaciones existentes entre la IE como rasgo y salud mental serán más elevadas que las relaciones entre IE como capacidad y salud mental.

H3: No existirán diferencias en función de la etapa educativa en la que actualmente ejercen los orientadores educativos participantes en ninguna de las variables estudiadas.

H4: Las mujeres mostrarán mayores puntuaciones que los hombres en las variables de IE como capacidad; por otro lado, los hombres mostrarán mayores puntuaciones que las mujeres en la variable de IE como rasgo.

H5: La salud mental tendrá como variable predictora la IE como rasgo.

\section{Método}

\section{Participantes}

Para este estudio se empleó un muestreo no probabilístico del tipo casual o por accesibilidad. La muestra ha estado compuesta de 203 profesionales orientadores en ejercicio pertenecientes en su totalidad a centros españoles de titularidad pública que desempeñan su trabajo en las distintas etapas educativas no universitarias: Educación Secundaria Obligatoria y Bachillerato (51,2\%), Educación Infantil y Primaria (48,8\%). En cuanto al sexo, el 45,3\% son mujeres, y el 54,7\% hombres. El rango de edad oscila entre 24 y 59 años, con una media de 35,35 años (DT= 8,25).

\section{Instrumentos}

Para evaluar la IE como capacidad se utilizó Mayer-Salovey-Caruso Emotional Intelligence Test (MSCEIT; Mayer, Salovey y Caruso, 2000). Es un instrumento de medida de la IE como capacidad basado en las ramas de IE de percepción, facilitación, comprensión y regulación. El MSCEIT evalúa cuatro factores correspondientes al modelo teórico de Mayer y Salovey (1997): percepción y expresión emocional, facilitación emocional, comprensión emocional y regulación de las emociones propias y de los demás. Además proporciona una 
puntuación total de inteligencia emocional como capacidad así como puntuaciones en dos áreas, experiencial y estratégica, y en cada una de las tareas específicas que incluye el test. La prueba presenta unas propiedades psicométricas adecuadas. La fiabilidad en la adaptación española, que informa el manual del instrumento, explicita que para la puntuación total es de .95 En cuanto a cada una de las ramas la fiabilidad ha sido la siguiente: (IECP) Percepción emocional (.93); (IECF) Facilitación emocional (.76); (IECC) Comprensión emocional (.83) y (IECR) Regulación emocional (.85).

Para evaluar la IE como rasgo se utilizó Trait Emotional Intelligence Questionnaire Short Form (TEIQUE-SF; Petrides y Furnham 2006; adaptación al castellano en la versión reducida por Pérez-González, 2010). Este instrumento incluye 30 ítems que evalúan las 15 subescalas que los autores señalan en su modelo del constructo de IE como rasgo: 1) Adaptabilidad; 2) Asertividad; 3) Percepción emocional; 4) Expresión emocional; 5) Dirección emocional de otros; 6) Autocontrol-Autorregulación emocional; 7) Estilo reflexivo (baja impulsividad); 8) Habilidades de mantenimiento de las relaciones; 9) Autoestima; 10) Automotivación; 11) Competencia social; 12) Tolerancia al estrés; 13) Empatía; 14) Felicidad; y 15) Optimismo. Los ítems que componen el cuestionario TEIQue-SF se puntúan en una escala formato tipo Likert de siete puntos (1=completamente en desacuerdo; 7=completamente de acuerdo). La puntuación global de la escala total se obtiene a través de la suma de los 30 ítems del cuestionario. En el presente estudio el instrumento obtuvo un índice de consistencia interna mediante alfa de Cronbach de .84 .

Para evaluar la salud mental se utilizó la Escala de Salud Mental (MH5; Ware y Sherbourne, 1992; adaptado al español por Alonso, Prieto y Antó, 1995). La Mental Health-5 (MH-5) es una de las subescalas del cuestionario de salud SF-36 de Ware y Sherbourne, (1992), adaptado al español por Alonso, Prieto y Antó (1995). El MH-5 está compuesta de 5 ítems del área de bienestar emocional y evalúa la salud mental de los sujetos, en concreto, el grado de sintomatología depresiva y ansiosa que ha presentado el sujeto durante el último mes se ha utilizado para evaluar el salud mental de los sujetos. Una puntuación elevada en esta escala se asocia con una mejor salud mental. Su carácter reducido la convierte en un instrumento muy útil para la investigación. Las preguntas que contiene esta escala son del tipo: “Durante las últimas cuatro semanas, ¿cuánto tiempo estuvo muy nervioso?” y las respuestas se codifican por medio de una escala tipo Likert que oscila desde $1=$ Siempre a $6=$ Nunca (Siempre, Casi siempre, Muchas veces, Algunas veces, Sólo alguna vez, Nunca). A los parti- 
cipantes se les pregunta cuestiones del tipo: "Durante las últimas cuatro semanas, ¿cuánto tiempo se sintió tan bajo de moral que nada podía animarle?". Sus propiedades psicométricas han mostrado una adecuada fiabilidad y validez a lo largo de más de una década de incesantes investigaciones. La fiabilidad en los estudios que han utilizado esta escala ha sido adecuada, con un alfa de Cronbach oscilante entre .77 y .85 (Vilagut, Ferrer, Rajmil, y Rebollo, 2005). En el presente estudio el instrumento obtuvo un índice de consistencia interna mediante alfa de Cronbach de .81 .

\section{Procedimiento}

La administración de los instrumentos se realizó durante el curso académico 2013/2014 en una sesión. Tenían un carácter anónimo y fueron cumplimentadas por los sujetos de la muestra en presencia del autor del artículo. El estudio cumplió con los valores éticos necesarios en la investigación con seres humanos (consentimiento informado y el derecho a la información, la protección de los datos personales y garantías de confidencialidad y de no discriminación).Además, todos los instrumentos se aplicaron siguiendo las instrucciones y las recomendaciones de los autores.

\section{Análisis Estadístico}

En primer lugar se realizó un estudio descriptivo de las respuestas dadas por los orientadores participantes a los instrumentos utilizados. A continuación se analizaron las correlaciones entre las puntuaciones correspondientes a todas las variables. Posteriormente se estudiaron las diferencias de medias ( $t$ de Student) en función de la etapa educativa en el que se encuentran ejerciendo actualmente los orientadores y en función del sexo de los participantes. Finalmente, con el objetivo de explorar las variables que predicen la salud mental, es decir, una alta puntuación en esta variable criterio, se realizó un análisis de regresión lineal múltiple, paso a paso.

\section{Resultados}

\section{Relación entre IE capacidad, IE como rasgo de personalidad y salud mental}

En la tabla 1 se muestran los estadísticos descriptivos y los resultados del análisis de correlación de Pearson de las medidas utilizadas en el estudio. Con respecto a la asociación de la salud mental con la IE como rasgo se relacionó de manera estadísticamente significativa y positiva $(r=.53 . p<.01)$. En cuanto a la asociación de la salud mental con la IE como capa- 
cidad se relacionó de manera estadísticamente significativa y positiva: con la puntuación global de IE como capacidad $(r=.29 . p<.01)$; con la puntuación de la IE como capacidad rama de facilitación emocional $(r=.20 . p<.05)$; y con la puntuación de la IE como capacidad rama de comprensión emocional $(r=.26 . p<.01)$. Por otro lado, no se encontraron asociaciones significativas en la puntuación de la IE como capacidad rama de percepción emocional y en la puntuación de la IE como capacidad rama de regulación emocional.

En cuanto a la asociación entre la puntuación global de IE como rasgo y las puntuaciones de IE como capacidad se relacionó de manera estadísticamente significativa y positiva con todas ellas, destacando la relación existente con la puntuación global de IE como capaci$\operatorname{dad}(r=.38 . p<.01)$.

Tabla 1. Matriz de correlaciones y estadísticos descriptivos de las variables de inteligencia emocional como capacidad, de inteligencia emocional como rasgo de personalidad y salud mental.

\begin{tabular}{cccccccccc}
\hline & 1 & 2 & 3 & 4 & 5 & 6 & 7 & Media & DT \\
\hline IECG & - & & & & & & & 99.17 & 10.98 \\
IECP & $.76^{* *}$ & - & & & & & & 100.48 & 12.89 \\
IECF & $.72^{* *}$ & $.49^{* *}$ & 1 & & & & & 95.94 & 9.90 \\
IECC & $.64^{* *}$ & $.23^{* *}$ & $.31^{* *}$ & - & & & & 102.72 & 12.05 \\
IECR & $.67^{* *}$ & $.28^{* *}$ & $.36^{* *}$ & $.37^{* *}$ & - & & & 98.51 & 11.51 \\
IERA & $.38^{* *}$ & $.35^{* *}$ & $.29^{* *}$ & $.25^{* *}$ & $.17^{*}$ & - & & 4.69 & .61 \\
AJPS & $.29^{* *}$ & .19 & $.20^{*}$ & $.26^{* *}$ & .15 & $.53^{* *}$ & - & 14.95 & 4.25 \\
\hline
\end{tabular}

Nota: (1) IECG: Puntuación global de IE como capacidad; IECP: Puntuación de la IE como capacidad rama de percepción emocional; IECF: Puntuación de la IE como capacidad rama de facilitación emocional; IECC: Puntuación de la IE como capacidad rama de comprensión emocional; IECR: Puntuación de la IE como capacidad rama de regulación emocional; IERA: Puntuación global de IE como rasgo; AJPS: Puntuación global de salud mental $(2)^{*}=p<.05 ; * *=p<.01$.

Diferencias de medias en función de la etapa educativa en el que se encuentra ejerciendo actualmente los orientadores participantes

Para explorar las posibles diferencias de medias en función de la etapa en la que los orientadores participantes están ejerciendo actualmente, se crearon dos subgrupos para su comparación mediante la prueba $t$ de Student para muestras independientes (ver tabla 2): submuestra de orientadores que están ejerciendo en Educación Infantil y Primaria ( $\mathrm{N}=99)$ y submuestra de orientadores que están ejerciendo en Educación Secundaria Obligatoria y Bachillerato $(\mathrm{N}=104)$. 
En cuanto a las variables de IE como capacidad se hallaron diferencias significativas en función de la etapa en la que los orientadores participantes están ejerciendo actualmente únicamente en la puntuación de la IE como capacidad rama de comprensión emocional. En este sentido se constatan puntuaciones significativamente más elevadas en la puntuación de la IE como capacidad rama de comprensión emocional en los orientadores de Educación Secundaria Obligatoria y Bachillerato. Para poder valorar la magnitud de estas diferencias se calculó el tamaño del efecto (Cohen, 1992) observándose un tamaño del efecto moderado $(d=.37)$.

En cuanto a la variable de IE como rasgo no se hallaron diferencias significativas en función de la etapa en la que los orientadores participantes están ejerciendo actualmente. En cuanto a la variable salud mental también se hallaron diferencias significativas en función de la etapa en la que los orientadores participantes están ejerciendo actualmente. En este sentido se constatan puntuaciones significativamente más elevadas en la variable salud mental en los orientadores de Educación Infantil y Primaria. Para poder valorar la magnitud de estas diferencias se calculó el tamaño del efecto observándose un tamaño del efecto moderado $(d=.47)$.

Tabla 2. Media, desviación típica (DT), $t$ de Student, y tamaño del efecto de las diferencias de medias (d) en función de la etapa en la que se encuentran ejerciendo actualmente los orientadores participantes

\begin{tabular}{ccccccc}
\hline \multicolumn{2}{c}{ Primaria } & \multicolumn{2}{c}{ Secundaria } & & \\
\hline Variables & Media & DT & Media & DT & $\mathrm{t}$ & $\mathrm{d}$ \\
IECG & 97.78 & 10.99 & 100.56 & 10.85 & -1.68 & \\
IECP & 100.08 & 13.21 & 100.88 & 12.62 & -.41 & \\
IECF & 95.00 & 9.75 & 96.88 & 10.01 & -1.26 & \\
IECC & 100.52 & 11.24 & 104.91 & 12.48 & $-2.45^{*}$ & .37 \\
IECR & 97.89 & 12.39 & 99.13 & 10.59 & -.71 & \\
IERA & 4.63 & .61 & 4.75 & .60 & -1.32 & \\
AJPS & 15.74 & 4.32 & 13.80 & 3.91 & $2.44^{*}$ & .47 \\
\hline
\end{tabular}

Nota: (1) IECG: Puntuación global de IE como capacidad; IECP: Puntuación de la IE como capacidad rama de percepción emocional; IECF: Puntuación de la IE como capacidad rama de facilitación emocional; IECC: Puntuación de la IE como capacidad rama de comprensión emocional; IECR: Puntuación de la IE como capacidad rama de regulación emocional; IERA: Puntuación global de IE como rasgo; AJPS: Puntuación global de salud mental (2) El valor de t corresponde al resultado de la prueba $t$ de Student en la que no se han asumido varianzas iguales; ${ }^{*}=p<.05 ; * *=p<.01 ; * * *=p<.001$; (3) Calculo del índice de Cohen (1992) o "diferencia media tipificada" para el tamaño del efecto de las diferencias para cada cada variable.

\section{Diferencias de medias en función del sexo de los orientadores participantes}

Para explorar las posibles diferencias de medias en función del sexo de los orientadores participantes. Para su comparación se utilizó la prueba $t$ de Student para muestras inde- 
pendientes (ver Tabla 3$)$ : submuestra de hombres $(\mathrm{N}=111)$ y submuestra de mujeres $(\mathrm{N}=$ 92).

En cuanto a las variables de IE como capacidad se hallaron diferencias significativas en función del sexo únicamente en la puntuación de la IE como capacidad rama de regulación emocional. En este sentido se constatan puntuaciones significativamente más elevadas en la puntuación de la IE como capacidad rama de regulación emocional en las mujeres. Para poder valorar la magnitud de estas diferencias se calculó el tamaño del efecto (Cohen, 1992) observándose un tamaño del efecto moderado $(d=.43)$.

En cuanto a la variable de IE como rasgo se hallaron diferencias significativas en función del sexo. En este sentido se constatan puntuaciones significativamente más elevadas en la puntuación de la IE como rasgo de personalidad en los hombres. Para poder valorar la magnitud de estas diferencias se calculó el tamaño del efecto observándose un tamaño del efecto pequeño $(d=.35)$.

En cuanto a la variable salud mental también se hallaron diferencias significativas en función del sexo. En este sentido se constatan puntuaciones significativamente más elevadas en la puntuación de salud mental en los hombres Para poder valorar la magnitud de estas diferencias se calculó el tamaño del efecto observándose un tamaño del efecto grande $(d=.71)$.

Tabla 3. Media, desviación típica (DT), t de Student, y tamaño del efecto de las diferencias de medias (d) en función del sexo de los orientadores participantes

\begin{tabular}{ccccccc}
\hline \multicolumn{2}{c}{ Hombres } & \multicolumn{2}{c}{ Mujeres } & & \\
\hline Variables & Media & DT & Media & DT & t & $\mathrm{d}$ \\
IECG & 97.75 & 11.03 & 100.00 & 10.91 & -1.31 & \\
IECP & 100.28 & 13.33 & 100.59 & 12.68 & -.15 & \\
IECF & 94.63 & 9.25 & 96.70 & 10.22 & -1.38 & \\
IECC & 102.68 & 11.69 & 102.74 & 12.31 & -.03 & \\
IECR & 95.45 & 11.42 & 100.30 & 11.24 & $-2.74^{* *}$ & .43 \\
IERA & 4.82 & .59 & 4.61 & .61 & $2.24 *$ & .35 \\
AJPS & 16.42 & 4.07 & 13.57 & 3.98 & $3.68^{* * *}$ & .71 \\
\hline
\end{tabular}

Nota: (1) IECG: Puntuación global de IE como capacidad; IECP: Puntuación de la IE como capacidad rama de percepción emocional; IECF: Puntuación de la IE como capacidad rama de facilitación emocional; IECC: Puntuación de la IE como capacidad rama de comprensión emocional; IECR: Puntuación de la IE como capacidad rama de regulación emocional; IERA: Puntuación global de IE como rasgo; AJPS: Puntuación global de salud mental (2) El valor de t corresponde al resultado de la prueba t de Student en la que no se han asumido varianzas iguales; *p<.05; ** $p<.01$; ***p<.001; (3) Calculo del índice de Cohen (1992) o "diferencia media tipificada" para el tamaño del efecto de las diferencias para cada cada variable. 


\section{Variables predictoras de salud mental}

Con el objetivo de explorar las variables que predicen el salud mental, es decir, una alta puntuación en esta variable criterio, se realizó un análisis de regresión lineal múltiple, paso a paso, cuyos resultados se presentan en la Tabla 4. Como se puede observar en la Tabla 4, del conjunto de las variables predictoras de la salud mental, sólamente una resultó estadísticamente significativas: la puntuación global de IE como rasgo $($ Beta $=.582)$. Siendo su poder explicativo medio, ya que esta variable predictora explica el $33.4 \%$ de la varianza.

Tabla 4. Análisis de regresión múltiple lineal para variables predictoras de la salud mental

\begin{tabular}{|c|c|c|}
\hline \multirow[t]{3}{*}{ Variable } & \multicolumn{2}{|c|}{ AJPS } \\
\hline & \multicolumn{2}{|c|}{$F_{(1,202)}=51.780 * * *, \mathrm{R}_{\text {adj }}^{2}=.332$} \\
\hline & Beta & $\mathrm{t}$ \\
\hline IERA & .582 & $7.916 * * *$ \\
\hline
\end{tabular}

\section{Discusión}

Los resultados de nuestro estudio aportan evidencias sobre la importancia que la IE posee a la hora de predecir la salud mental de los orientadores. En primer lugar, se ofrecen evidencias de la relación estadísticamente significativa, positiva y moderada entre la IE como rasgo y la IE como capacidad destacando la relación existente con la puntuación global de IE como capacidad. Nuestros resultados están en consonancia con los encontrados por otros trabajos realizados en población adulta y que evalúan la IE mediante pruebas de rendimiento típico y pruebas de rendimiento máximo en los que las correlaciones entre ambas puntuaciones tienden a encontrarse en torno a $r=.30$ (Furnham, 2001). Estos resultados confirman la hipótesis 1 que proponía que existirán relaciones moderadas entre IE como capacidad e IE como rasgo de personalidad.

Asimismo se ratifica la hipótesis 2 que postulaba que las relaciones existentes entre la IE como rasgo y la salud mental $(r=.53 . p<.01)$ serán más elevadas que las relaciones entre IE como capacidad y salud mental $(r=.29 . p<.01)$. Igualmente nuestros resultados se encuentran en la misma dirección que otros estudios (e.g., Bastian, Burns y Nettelbeck, 2005; 
Brackett, Mayer y Warner, 2004; Gardner y Qualter, 2009; Mavroveli, Petrides, Rieffe y Baker, 2007; Mikolajczak, Luminet y Menil, 2006; Mikolajczak, Luminet, Leroy y Roy, 2007; Petrides, Pérez-González y Furnham, 2007; Petrides, Pita y Kokkinaki, 2007) recogidos en el metaanálisis de Martins, Ramalho y Morin (2010) que concluye que la asociación promedio de la IE como capacidad y la IE como rasgo con la salud mental es de $(\bar{r}=.36)$.

Por otro lado, los hallazgos confirman parcialmente la hipótesis 3, ya que existen diferencias en función de la etapa educativa en la que actualmente ejercen los orientadores educativos participantes en la variable de IE como capacidad concretamente se hallaron diferencias significativas en la puntuación de la IE como capacidad rama de comprensión emocional se constatan puntuaciones significativamente más elevadas en los orientadores de Educación Secundaria Obligatoria y Bachillerato. Probablemente los orientadores de Educación Secundaria debido a las características psicoevolutivas de los adolescentes tengan mayor necesidad de comprender el estilo emocional, la forma de actuar de sus alumnos, y necesiten interpretar el significado de las emociones complejas ya que están sujetos a un elevado número de situaciones interpersonales así como una búsqueda de la identidad personal y, obviamente tengan que ofrecer un mayor apoyo. También se constatan puntuaciones significativamente más elevadas en la variable salud mental en los orientadores de Educación Infantil y Primaria. Una posible explicación de estos resultados están relacionados con otros estudios en los que los profesores de Educación Infantil y Primaria obtienen menores puntuaciones en burnout en comparación con los profesores de Educación Secundaria Obligatoria y Bachillerato (e.g., Salanova, Llorens y García-Renedo, 2003).

De igual modo, los resultados obtenidos son coherentes con los obtenidos en otros estudios sobre las diferencias en IE como capacidad y IE como rasgo en función del sexo (e.g., Extremera, Fernández-Berrocal, y Salovey, 2006; Sánchez-Núñez, Fernández-Berrocal, Montañés y Latorre, 2008). En este sentido la mayoría de las investigaciones han mostrado que las mujeres tienden a presentar mayores niveles de IE como capacidad siendo el tamaño del efecto mediante $d$ de Cohen $(d=.47)$ tal y como recoge el metaanálisis de Joseph y Newman (2010). En nuestro estudio se constatan puntuaciones significativamente más elevadas en las mujeres en la puntuación de la IE como capacidad exclusivamente en la rama de regulación emocional siendo similar el tamaño del efecto mediante $d$ de Cohen $(d=.43)$ Por otra parte, los estudios que se han centrado en las diferencias sexuales en la IE como rasgo presentan grandes controversias. Algunos de estos estudios revelan que los hombres tienden a presentar ni- 
veles mayores mediante pruebas de rendimiento típico que las mujeres (Brackett y Mayer, 2003; Petrides y Furnham, 2000). Sin embargo, otros estudios concluyen que no existen tales diferencias (e.g., Joseph y Newman, 2010). En nuestro estudio se constata puntuaciones significativamente más elevadas en la puntuación de la IE como rasgo de personalidad en los hombres si bien, es cierto que se observa un tamaño del efecto pequeño $(d=.35)$. Confirmándose así la hipótesis 4.

Por último los hallazgos confirman la hipótesis 5, ya que el análisis de regresión múltiple ha permitido identificar como variable predictora de la salud mental, la IE como rasgo. Estos resultados están en consonancia con los recogidos en el metaanálisis de Martins, Ramalho y Morin (2010) que concluye que la variable IE como rasgo moderaba la relación con la salud mental en mayor medida que IE como capacidad.

En definitiva, nuestro estudio ha supuesto uno de los primeros trabajos llevados a cabo en una muestra española de orientadores educativos en los que se analiza, de manera combinada, la influencia de la IE como rasgo medida a través de pruebas de rendimiento típico y la IE como capacidad medida a través de pruebas de rendimiento máximo, sobre un indicador de salud mental. Además aporta evidencias de validez predictiva de la IE como rasgo sobre un indicador de salud mental. Futuras investigaciones deberán continuar esta línea de investigación corroborando los resultados aquí encontrados a través de diseños prospectivos que permitan inferir relaciones de causalidad entre las variables estudiadas y examinando la influencia de la IE como rasgo y como capacidad sobre otras variables criterio importantes en el colectivo de orientadores educativos, como el burnout, el nivel de satisfacción laboral o el rendimiento en el trabajo. Por otra parte, el avance en la investigación de la IE en general necesita de un esfuerzo de los investigadores por diseñar otras medidas de rendimiento máximo que permitan evaluar la IE como capacidad y que demuestren su validez en diversas poblaciones.

A pesar de estos hallazgos, nuestra investigación presenta una serie de limitaciones que es necesario subrayar. La primera de las limitaciones hace referencia al carácter no aleatorio de la muestra empleada, que limita su representatividad y, por tanto, debemos ser precavidos a la hora de generalizar estos resultados. La segunda de las limitaciones se refiere a que la muestra pertenece exclusivamente a una comunidad autónoma y no sabemos si en otro marco sociocultural o un incremento en el tamaño de la muestra modificaría los resultados. Para subsanar estas limitaciones el equipo investigador tiene en perspectiva llevar a cabo una amplia- 
ción de este estudio contando con una muestra en la que participen orientadores en ejercicio de varias comunidades autónomas.

A pesar de estas limitaciones, los resultados del trabajo tienen implicaciones prácticas para la formación inicial y continua de los orientadores educativos ya que puede proporcionar evidencias a favor de la importancia de las competencias sociopersonales en el desarrollo de las funciones, responsabilidades y tareas que estos profesionales llevan a cabo en el contexto escolar. Igualmente estos resultados conllevan implicaciones en el campo de la intervención y la prevención psicológica ya que pueden ayudar a identificar déficits en aspectos específicos de la IE como capacidad y de la IE como rasgo que supongan una mejora en la salud mental en el colectivo de orientadores educativos. 


\section{Referencias}

Alonso, J., Prieto, L., \& Antó, J. M. (1995). La versión española del SF-36 Health Survey (Cuestionario de Salud SF-36): un instrumento para la medida de los resultados clínicos. Medicina Clínica, 104, 771-776.

Bar-On, R. (2000). Emotional and social intelligence: insights from emotional quotient inventory. En Bar-On, R. \& J.D.A. Parker (Eds.). The handbook of emotional intelligence: Theory, development, assessment, and application at home, school, and in the workplace (pp. 343-362). San Francisco: Jossey-Bass.

Bastian, V. A., Burns, N. R., \& Nettelbeck, T. (2005). Emotional intelligence predicts life skills, but not as well as personality and cognitive abilities. Personality and Individual Differences, 39, 1135-1145. doi:10.1016/j.paid.2005.04.006

Bisquerra, R. (2006). Orientación psicopedagógica y educación emocional. Estudios sobre Educación, 11, 9-25.

Bisquerra, R., \& Pérez, N. (2007). Las competencias emocionales. Educación XXI, 10, 61-82.

Bisquerra, R., Pérez-González, J.C \& García, E. (2015). Inteligencia emocional en educación. Madrid: Síntesis.

Brackett, M. A., \& Mayer, J. D. (2003). Convergent, discriminant, and incremental validity of competing measures of emotional intelligence. Personality and Social Psychology Bulletin, 29, 1147-1158.doi: 10.1177/0146167203254596

Brackett, M. A., Mayer, J. D., \& Warner, R. M. (2004). Emotional intelligence and its relation to everyday behaviour. Personality and Individual Differences, 36, 1387-1402. doi:10.1016/S0191-8869(03)00236-8

Cohen, J. (1992). A Power Primer. Psychological Bulletin, 112, 155-159. doi:10.1037/00332909.112.1.155

Cronbach, L. J. (1972). Fundamentos de la exploración psicológica. Madrid: Biblioteca nueva.

Davies, M., Syankov, L., \& Roberts, R. (1998). Emotional intelligence: In search of elusive construct. Journal of Personality and Social Psychology, 75, 989-1015. http://dx.doi.org/10.1037/0022-3514.75.4.989 
Extremera, N., \& Fernández-Berrocal, P. (2004). El papel de la inteligencia emocional en el alumnado: evidencias empíricas. Revista Electrónica de Investigación Educativa, 6 (2), 1-17.

Extremera, N., Fernández-Berrocal, P., \& Salovey, P. (2006). Spanish version of the MayerSalovey-Caruso Emotional Intelligence Test (MSCEIT) version 2.0: reliabilities, age and gender differences. Psicothema, 18 (Suppl), 42-48.

Fernández-Berrocal, P., \& Extremera, N. (2006). La investigación de la Inteligencia Emocional en España. Ansiedad y Estrés, 12, 139-153.

Furnham, A. (2001). Self-estimates of intelligence: Culture and gender difference in self and other estimates of both general (g) and multiple intelligences. Personality and Individual Differences, 31, 1381-1405. doi:10.1016/S0191-8869(00)00232-4

Gardner, K., \& Qualter, P. (2009). Emotional intelligence and borderline personality disorder. Personality and Individual Differences, 47, 94-98. doi:10.1016/j.paid.2009.02.004

Goleman, D. (1995). Emotional Intelligence: Why it can matter more than IQ. New York: Bantam Books.

Grañeras, M., \& Parras, A. (2008). Orientación educativa: Fundamentos teóricos, modelos institucionales y nuevas perspectivas. Ministerio de Educación, Política Social y Deporte. CIDE: Madrid.

Joseph, D. L., \& Newman, D. A. (2010). Emotional intelligence: An integrative meta-analysis and cascading model. Journal of Applied Psychology, 95, 54-78. doi: $10.1037 / \mathrm{a} 0017286$

Malouff, J. M., Schutte, N. S., \& Thorsteinsson, E. B. (2012). A meta-analysis of the relationship between emotional intelligence and romantic relationship satisfaction. Manuscript submitted for publication.

Martínez, Mª C., Quintanal, J., \& Téllez, J. A. (2002). La Orientación Escolar. Fundamentos y Desarrollo. Madrid: Dykinson.

Martínez-González, A. E., Piqueras, J.A., \& Ramos-Linares, V. (2010). Emotional Intelligence in physical and mental health. Electronic Journal of Research in Educational Psychology, 8(2), 861- 890.

Martins, A., Ramalho, N., \& Morin E. (2010). A comprehensive meta-analysis of the relationship between emotional intelligence and health. Personality and Individual Differences, 49, 554-564. doi:10.1016/j.paid.2010.05.029

Mavroveli, S., Petrides, K. V., Rieffe, C., \& Bakker, F. (2007). Trait emotional intelligence, psychological well-being and peer-rated social competence in adolescence. British 
Journal of Developmental Psychology,25(2), 263-275. doi: 10.1348/026151006X118577

Mayer, J., Caruso, D., \& Salovey, P. (2000). Models of emotional intelligence. In R. Sternberg (Ed.), Handbook of intelligence (pp. 396-420). New York, USA: Cambridge University Press.

Mayer, J., \& Salovey, P. (1997). What is emotional intelligence. In P.Salovey y D. Sluyter (Eds.), Emotional development and emotional intelligence: educational implications (pp. 3-34). New York, USA: Basic Books.

Mayer, J. D., Salovey, P., \& Caruso, D. R. (2002). Mayer-Salovey-Caruso Emotional Intelligence Test (MSCEIT) user's manual. Toronto, ON: Multi-Health Systems.

Mikolajczak, M., Luminet, O., \& Menil, C. (2006). Predicting resistance to stress: Incremental validity of trait emotional intelligence over alexithymia and optimism. Psicothema, 18(Suppl), 79-88.

Mikolajczak, M., Luminet, O., Leroy, C., \& Roy, E. (2007). Psychometric properties of the trait emotional intelligence questionnaire: Factor structure, reliability, construct, and incremental validity in a French-speaking population. Journal of Personality Assessment, 88(3), 338-353.

O'Boyle, E. H., Humphrey, R. H., Polack, J. M., Hawver, T. H., \& Story, P. A. (2011). The relation between emotional intelligence and job performance: A meta-analysis. Journal of Organizational Behavior, 32(5), 788-818. doi: 10.1002/job.714

O'Connor, R. M., \& Little, I. S. (2003). Revisiting the predictive validity of emotional intelligence: self-report versus ability-based measures. Personality and Individual Differences, 35(8), 1893-1902. doi: http://dx.doi.org/10.1016/S0191-8869\%2803\%29000382

Pérez-González, J. C. (2010). Revisión del sentido y de los contenidos de la orientación personal. Revista Española de Orientación y Psicopedagogía (REOP), 21(2), 434-442.

Pérez-González, J.C., Petrides, K.V., \& Furnham, A. (2007). La medida de la inteligencia emocional rasgo. En J. M. Mestre y P. Fernández-Berrocal, Manual de Inteligencia Emocional (pp. 81-97). Madrid: Pirámide.

Petrides, K.V. (2011). Ability and trait emotional intelligence. In T. Chamorro-Premuzic, A. Furnham, S. von Stumm (Eds.), The Blackwell-Wiley handbook of individual differences. New York: Wiley. 
Petrides, K. V., Frederickson, N., \& Furnham, A. (2004). The role of trait emotional intelligence in academic performance and deviant behavior at school. Personality and Individual Differences, 36, 277-293. doi:10.1016/S0191-8869(03)00084-9

Petrides, K. V., \& Furnham, A. (2000). On the dimensional structure of emotional intelligence. Personality and Individual Differences, 29, 313- 320.

Petrides, K. V., \& Furnham, A. (2001). Trait emotional Intelligence: Psychometric Investigation with Reference to Established Trait Taxonomies. European Journal of Personality, 15, 425-448. doi: 10.1002/per.416

Petrides, K. V., \& Furnham, A. (2003). Trait emotional intelligence: Behavioral validation in two studies of emotion recognition and reactivity to mood induction. European Journal of Personality, 17, 39-57. doi: 10.1002/per.466

Petrides, K. V. \& Furnham, A. (2006). The role of trait emotional intelligence in a genderspecific model of organizational variables. Journal of Applied Social Psychology, 36 (2), 552-569.

Petrides, K. V., Pérez-González, J. C., \& Furnham, A. (2007). On the criterion and incremental validity of trait emotional intelligence. Cognition and Emotion, 21(1), 26-55. doi: $10.1080 / 02699930601038912$

Petrides, K. V., Pita, R., \& Kokkinaki, F. (2007). The location of trait emotional intelligence in personality factor space. British Journal of Psychology, 98, 273-289. doi:10.1348/000712606X120618

Roberts, R. D., Zeidner, M. \& Matthews, G. (2007). Emotional intelligence. Knowns and unknowns. En G. Matthews, M. Zeidner \& R. D. Roberts (Eds.). The science of emotional intelligence: Knowns and Unknowns (pp. 419-474). Oxford: Oxford University Press.

Rodríguez-Moreno, M. L. (1995). Orientación e intervención psicopedagógica. Barcelona: CEAC.

Salanova, M., Llorens, S., \& García-Renedo, M. (2003). ¿Por qué se están ‘quemando’ los profesores? Revista de Prevención, Trabajo y Salud, 28, 16-20.

Salovey, P., \& Mayer, J. D. (1990). Emotional intelligence. Imagination, cognition and personality, 9, 185-211. doi:0.2190/DUGG-P24E-52WK-6CDG

Sánchez-Núñez, M.T, Fernández-Berrocal, P., Montañés, J., \& Latorre, J.M (2008). ¿Es la inteligencia emocional una cuestión de género? Socialización de las competencias emocionales en hombres y mujeres y sus implicaciones. Electronic Journal of Research in Educational Psychology, 6(2), 455-474. 
Schutte, N. S., Malouff, J. M., Thorsteinsson, E. B., Bhullar, N., \& Rooke, S. E., (2007). A meta-analytic investigation of the relationship between emotional intelligence and health. Personality and Individual Differences, 42, 921-933. doi: 10.1016/j.paid.2006.09.003

Schutte, N., Malouff, J., \& Thorsteinsson, E. (2013). Increasing Emotional Intelligence through Training: Current Status and Future Directions. The International Journal of Emotional Education, 5(1), 56-72.

Vilagut G, Ferrer M, Rajmil L, Rebollo P, Permanyer-Miralda G., \& Quintana J.M. (2005). El cuestionario de salud SF-36 español: una década de experiencia y nuevos desarrollos. Gaceta Sanitaria, 19, 135-150. doi:10.1157/13074369

Ware, J.E., \& Sherbourne, C.D. (1992). The MOS 36-item short-form health survey (SF-36). Medical Care, 30(6), 473-483. doi: 10.1161/01.STR.27.10.1812 